Background Management of postoperative pain is a subject of interest as we believe that pain is still inadequately relieved in this population.

Purpose To describe methods of postoperative pain management in anaesthesia-resuscitation and surgery services of Mohammed V Military Teaching Hospital in Rabat.

Materials and Methods A questionnaire was distributed to our hospital anaesthesia-resuscitation doctors and surgeons. The questionnaire was designed to explore the evaluation, treatment and provision of postoperative pain prevention.

Results 27 answers (78\%) were obtained. 9 services stated that this was making them aware of the problem of postoperative pain management. $81.5 \%$ of the professionals didn't have a written protocol. Postoperative pain was only evaluated in $32 \%$ of the patients. Among the methods used for postoperative pain measurement in post-surgical care units, simple verbal scales were the most used by professionals (29.6\%), followed by an analogue visual scale (25.9\%). Paracetamol was the drug most used in pain treatment.

Conclusions Although our investigation generated fairly satisfactory results, our hospital professionals must give greater importance to postoperative pain management in order to improve their patients' pain relief.

No conflict of interest.

\section{CPC-081 MANAGEMENT OF SEVERE ANAEMIA WITH RECOMBINANT HUMAN ERYTHROPOIETIN IN A JEHOVAH'S WITNESS PATIENT: CASE REPORT AND REVIEW OF LITERATURE}

doi:10.1136/ejhpharm-2013-000276.538

'J Giraud, ' $\mathrm{J}$ Jezequel, ${ }^{2} \mathrm{~K}$ Abdel Aal, ${ }^{1} \mathrm{~V}$ Duperrin, ${ }^{2 \mathrm{R}}$ Geha, ${ }^{1} \mathrm{~A}$ Fabreguettes. ${ }^{1} \mathrm{CH}$ Robert Ballanger, Pharmacy, Aulnay sous Bois, France; ${ }^{2} \mathrm{CH}$ Robert Ballanger, Cardiology, Aulnay Sous Bois, France

Background The medical care of Jehovah's Witness patients, because they refuse blood transfusion, becomes problematic in cases of severe life-threatening anaemia.

Purpose To describe the case of a patient with severe anaemia who received erythropoietin (EPO) treatment as the result of a literature review.

Materials and Methods A 77-year old woman was sent to the emergency department with thoracoepigastric pain, blood clots and vomiting for a week. Cardiac examination revealed a coronary syndrome caused by gastrointestinally-induced anaemia at $5.6 \mathrm{~g} / \mathrm{dl}$ (haematocrit $=18.9 \%$ ). On day 3 the haemoglobin fell to $4 \mathrm{~g} / \mathrm{dl}$ (haematocrit $=14.4 \%$ ) upon which a treatment with EPO beta at 30,000 IU per week (380 IU/kg/week) associated with high intravenous iron supplementation (300 mg/48 hours) was instituted. After 16 days of treatment haemoglobin $(8.9 \mathrm{~g} / \mathrm{dl})$ and haematocrit $(31.6 \%)$ had doubled and clinical improvement was observed. The patient was discharged on day 22 of treatment with a total of 4 EPO injections (haemoglobin $=9.6 \mathrm{~g} / \mathrm{dl}$ ).

Results Currently in emergency there is no alternative to transfusion and a higher mortality is linked to a low haemoglobin level. In a multicentre study with 148 patients, Georgopoulos et al, showed the efficacy of EPO, used off-label, administered once weekly, to reduce transfusions.

Thirteen recent publications reported experiences with the intravenous or subcutaneous administration of EPO in anaemia treatment. The optimal dose of EPO remains unclear: dosage ranges from $200 \mu \mathrm{g} /$ week darbepoetin alfa (Gutierrez et al), to $130 \mathrm{IU} / \mathrm{kg}$ of EPO three times weekly (Walton et al), to $600 \mathrm{Iu} / \mathrm{kg} /$ day for 2 days to $300 \mathrm{IU} / \mathrm{kg} /$ day (Cothren et al). After starting treatment the haemoglobin level doubled in 19 days (in an average of 4 days30 days).
Conclusions Our weekly EPO protocol is in the lower targets found in the literature but it appears as effective as other protocols. Significant variability without a major difference in efficacy appears when EPO is used for Jehovah's Witness patients, but EPO may provide an alternative treatment in life-threatening anaemia, when blood transfusions are not accepted.

No conflict of interest.

\section{CPC-082 MEASURING EFFECTIVITY: PHARMACEUTICAL INTERVENTIONS THROUGH COMPUTERISED PHYSICIAN ORDER ENTRY VERSUS DIRECT PHONE CALLS}

doi:10.1136/ejhpharm-2013-000276.539

I Aguirre Zubia, G Lizeaga Cundin, MJ Gayan Lera, MC Leunda Eizmendi, MC Andueza Granados, MA Aranguren Redondo, M Barrenetxea Zabala. Hospital Universitario Donostia, Pharmacy Service, San Sebastián, Spain

Background Computerized physician order entry (CPOE) implementation in hospitals has become an important tool for interactive validation of medical orders as well as a facilitator for pharmacist interventions. However several studies have investigated the 'alert fatigue' phenomenon caused by an elevated number or recommendations which can lead to relevant clinical interventions being bypassed.

Purpose To compare the degree of acceptance of pharmacist's interventions after medical order validation using CPOE versus direct phone conversation with the physician.

Materials and Methods Observational, descriptive and prospective study from May to August 2012

The intervention chosen for comparing the systems was FDA recommendation for simvastatin use regarding contraindications and maximum recommended doses. Interventions were generated using a quasi-random allocation method and physicians could refuse recommendations.

When an intervention assigned to the telephone call group was not possible, CPOE was used as a second option. Acceptance of recommendations and time to modifications of the prescriptions were recorded.

Results Phone call: only 34 of 42 attempted interventions were possible due to the prescriber's unavailability.

CPOE: 46 interventions and 54 interventions in total after the first attempt by phone call.

Rate of recommendations accepted was $82 \%$ for phone calls while only $52 \%$ of CPOE interventions.

Time to medical order modification since intervention was 0.26 days for the phone call group versus 2.18 days for CPOE group Conclusions $\mathrm{CPOE}$ is a useful tool for pharmacists to communicate with the multidisciplinary patient care team but when a relevant clinical intervention is necessary direct phone calls to prescribers are more effective and quicker.

Abstract CPC-082 Table 1

\begin{tabular}{llllll}
\hline $\begin{array}{l}\text { Concomitant } \\
\text { drug }\end{array}$ & $\begin{array}{l}\text { Number of } \\
\text { patients }\end{array}$ & $\begin{array}{l}\text { Number of } \\
\text { alerts made } \\
\text { by CPOE }\end{array}$ & $\begin{array}{l}\text { Recommen- } \\
\text { dations } \\
\text { accepted }\end{array}$ & $\begin{array}{l}\text { Number of } \\
\text { alerts made } \\
\text { by phone }\end{array}$ & $\begin{array}{l}\text { Recommen- } \\
\text { dations } \\
\text { accepted }\end{array}$ \\
\hline Amiodarone & 25 & 16 & $6(37 \%)$ & 9 & $9(100 \%)$ \\
Amlodipine & 26 & 15 & $9(60 \%)$ & 11 & $7(64 \%)$ \\
Ciclosporin & 1 & 1 & $1(100 \%)$ & - & - \\
Diltiazem & 26 & 15 & $9(60 \%)$ & 11 & $10(91 \%)$ \\
Gemfibrozil & 2 & 1 & $1(100 \%)$ & 1 & $1(100 \%)$ \\
Ketoconazole & 1 & 1 & $1(100 \%)$ & - & - \\
Verapamil & 7 & 5 & $1(20 \%)$ & 2 & $1(50 \%)$ \\
Total & $\mathbf{8 7}$ & $\mathbf{5 4}$ & $\mathbf{2 8}$ & $\mathbf{3 4}$ & $\mathbf{2 8}$ \\
Ratio & & $\mathbf{6 2} \%$ & $\mathbf{5 2} \%$ & $\mathbf{3 9} \%$ & $\mathbf{8 2} \%$ \\
\hline
\end{tabular}

No conflict of interest. 